\title{
Study of the two-photon hadronic events at very low $Q^{2}$ at LEP2
}

\author{
N. Zimin
}

JINR, Dubna and Dep. of Physics, University of Lund

\begin{abstract}
Results of an experimental study of two-photon hadronic events at very low momentum transfer $Q^{2}$ at LEP2 are presented. The data correspond to an integrated luminosity of about $146 \mathrm{pb}^{-1}$, collected by the DELPHI detector during the 1998 LEP run at centre-of-mass energies of $189 \mathrm{GeV}$. The DELPHI Very Small Angle Tagger is used to measure scattered electrons and positrons. Experimental distributions compared with Monte Carlo predictions are presented.
\end{abstract}

Talk given at the PHOTON 99 - International Conference on the Structure and Interactions of the Photon, Freiburg, Germany, 23-27 May 1999. 


\section{STATISTICS USED}

Studies of two-photon interactions at very low momentum transfer $Q^{2}$ (below $1 \mathrm{GeV}^{2} / c^{2}$ ) at LEP2, when both scattered $e^{+}$and $e^{-}$are detected, can provide new measurements of the total $\gamma \gamma$ cross-section and other interesting results $[1,2]$. The only way to study such events is to use the so-called very forward luminometers to tag scattered $e^{+}$and $e^{-}$. The upgrade for the smaller beam pipe proposed by the DELPHI collab. at the position of very forward calorimeters was carried out during the 1997-1998 shutdown. It yielded a factor $\simeq 2$ gain in statistics. The first results obtained at LEP2 for double tagged $\gamma \gamma$ events at very low $Q^{2}$ are presented here. All data collected by DELPHI in 1998 were analyzed in this study. After applying of certain quality criteria to operation of the subdetectors relevant to our analysis, the integrated luminosity was about $146 \mathrm{pb}^{-1}$. A two-photon event generator TWOGAM [3] describes DELPHI data reasonably well for the no-tag [4], single tag [5] and double[6] tag data at LEP1. The three component model is used in this generator to describe multihadron production: the existing generalized Vector meson Dominance Model (VDM) and Quark Parton Model (QPM) and the more recent Resolved Photon Contribution (RPC). More details about the model and parameters used for generation (as in previous studies) can be found in $[5,6]$. The Gordon-Storrow parametrization (GS2) of parton density functions in the photon is used to generate the RPC with $p_{t}^{\min }(G S 2)=1.88 \pm 0.020 \mathrm{GeV} / \mathrm{c}$, the transverse momentum of outgoing partons, a single free parameter found in our previous studies. Generation was performed separately for the VDM (the luminosity is $\left.270 \mathrm{pb}^{-1}\right) \mathrm{QPM},\left(660 \mathrm{pb}^{-1}\right)$ and RPC $\left(325 \mathrm{pb}^{-1}\right)$. The events generated were then fragmented using the JETSET 7.4 model and passed through the DELPHI simulation program and the same reconstruction program as for real data.

\section{DELPHI DETECTOR}

The DELPHI detector is described in detail in [7]. Only those subdetectors relevant to the present study are touched on in the present brief description. Scattered electrons were tagged by the Very Small Angle Tagger (VSAT) [8], a luminosity monitor consisting of four rectangular electromagnetic calorimeter modules placed horizontally on both sides of the beam pipe at $\pm 7.7 \mathrm{~m}$ from the interaction point. Each module $(5 \mathrm{~cm}$ high and $3 \mathrm{~cm}$ wide) consists of 12 tungsten absorbers (each two radiation lengths thick) interspersed by silicon planes, giving an energy reconstruction of $35 \% / \sqrt{E(G e V)}$. There are three silicon-strip planes placed around the longitudinal maximum of the electromagnetic showers: two measuring the horizontal position, and one the vertical position, giving a reconstructed position resolution of $200 \mu \mathrm{m}$. The modules are placed after the LEP superconducting quadrupole magnets, resulting in scattered leptons being focussed vertically and defocussed horizontally. The magnitude of this effect is inversely proportional to the lepton energy, resulting in a VSAT polar angle coverage from 4 to $14 \mathrm{mrad}$ for the $\gamma \gamma$ case. Following the upgrade proposed by DELPHI all LEP experiments were equipped with a smaller beam pipe at the position of their very forward calorimeters during the 1997-1998 shutdown. In the case of DELPHI the upgraded beam pipe has a $\simeq 0.5 \mathrm{~cm}$ smaller radius in the $x$-direction, and the VSAT modules have been moved closer to the beam line. Thus, the active detector area was enlarged together with an increase in cross-sections. 
The detector elements used for tracking through the uniform axial magnetic field were the Vertex Detector (VD), the Inner Detector (ID), the Time projection Chamber (TPC), the Outer Detector (OD), and the Forward Chambers in the endcap regions. Combining the information from the tracking detectors, the momentum resolution $\sigma_{p} / p$ varies from $0.001 \times p$ to $0.01 \times p(p$ in $\mathrm{GeV} / c)$, depending on the polar angle of the charged particle.

Electromagnetic energy is measured in the barrel region by the High density Projection Chamber (HPC) and in the forward and backward regions by a Forward Electro-Magnetic Calorimeter (FEMC). The Small angle Tile Calorimeter (STIC) is also used to cover the polar angles from from $2^{\circ}$ to the low edge of the FEMC.

Hadron shower energies are measured by combining the measurements from the HAdron Calorimeter (HAC) and electromagnetic calorimeters. Thus, all the DELPHI calorimeters are used in this analysis.

\section{EVENT SELECTION}

The very hard background conditions led us to use all natural criteria to select hadronic $\gamma \gamma$ events. Charged particles were selected as follows. The momentum had to be greater than $0.3 \mathrm{GeV} / c$ with a relative error less than 1 and the polar angle relative to the beam axis between $15^{\circ}$ and $165^{\circ}$. Hadronic events were selected by demanding three or more charged particles. Double tagged events had to have a measured energy $E_{t a g}>20 \mathrm{GeV}$ in both the forward and backward VSAT modules and no additional clusters with energy exceeding $20 \mathrm{GeV}$ in the STIC and FEMC. To reject distribution tails which exist in the data but not in the simulated events, these requirements were applied on the total energy of charged particles $(<25 \mathrm{GeV})$, total energy of neutral particles $(<20 \mathrm{GeV})$, total hadronic energy $(<40 \mathrm{GeV})$, total tagged and hadronic energy $(<200 \mathrm{GeV})$, longitudinal momentum $\left(P_{z}<25 \mathrm{Gev} / c\right)$ and transverse momentum $\left(P_{T}<8 \mathrm{Gev} / c\right)$ of the hadronic system. Similar criteria (found from the data and simulation comparison) were used both for charged and neutral components of the event and for combinations of total hadronic and tagged components.

Some of the distributions used to define selection criteria are shown in Fig. 1-3.

\section{BACKGROUND REJECTION}

Efficient rejection of the background is one of the major problems in this analysis. Independent studies performed by SL Division [9] at the DELPHI interaction point demonstrate that background conditions are now worse than those at LEP1. Using LEP luminosity monitors they found that the background event rate to the Bhabha events almost doubles with the energy increased up to $91.5 \mathrm{GeV}$. For double-tagged $\gamma \gamma$ events the main source of background comes from random coincidences between two independent events: Bhabha or off-momentum electrons hit the VSAT modules while the hadronic system comes from untagged or single tagged $\gamma \gamma$ events. Intensive studies were performed to eliminate background as much as possible. It was found that at LEP2 energies the situation is more complicated than at LEP1. In addition to the background from normal Bhabha events, two new sources appeared. Thus, in 1998 there were three main components of background, with different intrinsic features: a) normal Bhabha events which can be sufficiently rejected by the procedure developed previously [6], applying specific 


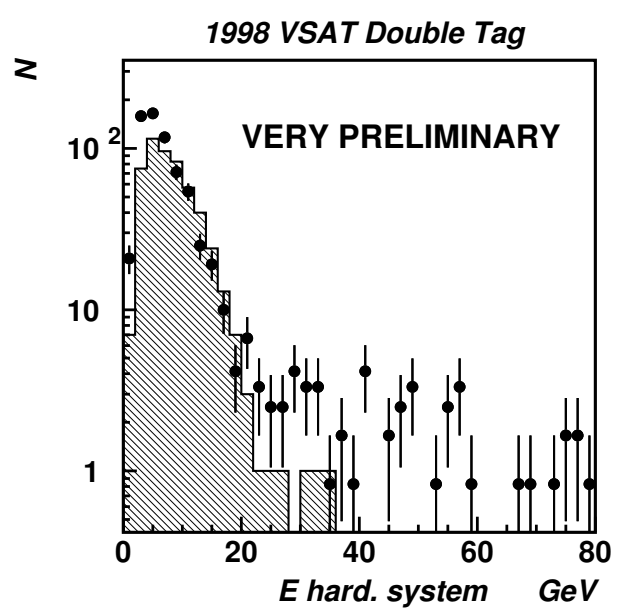

Figure 1: Energy of the hadronic system measured. Points are the data and the histogram is Monte Carlo prediction.

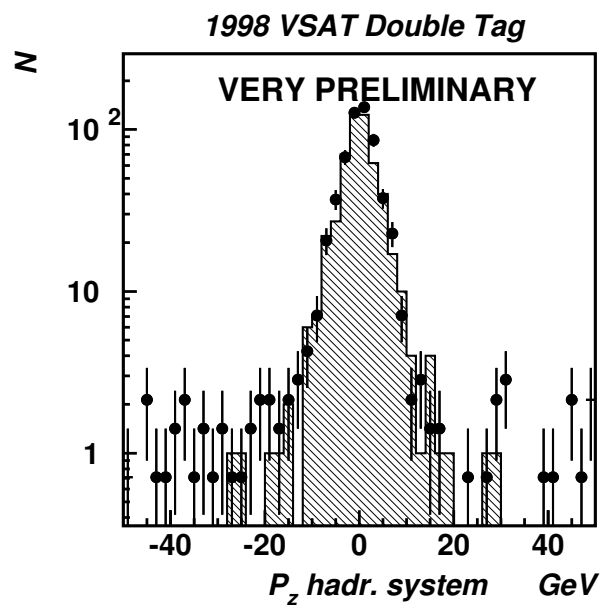

Figure 2: Longitudinal momentum of the hadronic system measured. Points are the data and the histogram is Monte Carlo prediction. 


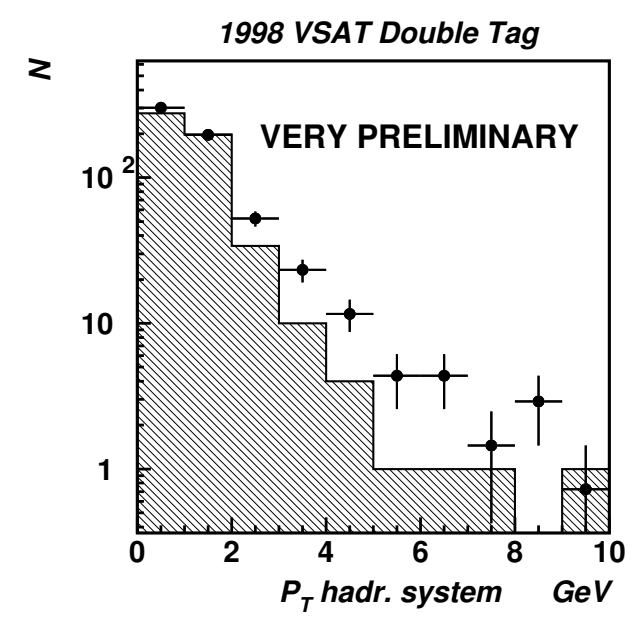

Figure 3: Transverse momentum of the hadronic system measured. Points are the data and the histogram is Monte Carlo prediction.

cuts on the difference in $\mathrm{X}$ and $\mathrm{Y}$ coordinates measured for both scattered leptons, which must be $\leq 2 \times \sigma$ of the corresponding narrow distributions found for each run, and on the energy measured in each module, which must be $\leq 0.7 \times$ Ebeam (Fig. 4); b) so-called "flange" Bhabhas, events with the same distributions for the difference in X and Y but pass the energy cut because they lose some energy in the flange in front of the modules: specific cuts on X coordinates are applied to reject them (Fig. 5); c) so-called "false" Bhabha events from the random coincidence of two off-momentum electrons hitting the VSAT opposite modules: specific cuts are applied on Y coordinates using the fact that they are located narrowly in the horizontal plane, the average position in $\mathrm{Y}$ was defined for each run when the off-momentum electrons were studied exploring the Bhabha event sample (Fig. 6). After all rejection criteria are applied the residual background was found to be $\simeq 12 \%$ in the final $\gamma \gamma$ sample. To calculate this probability, the random coincidences among the signals in the VSAT were studied for independent samples of cosmic muons, dimuons from $Z^{\circ}$ decays and Bhabha events measured by the VSAT and STIC.

\section{RESULTS}

Since the quality of the results mainly depends on the measurements of the tagged particles, all possible corrections were defined and applied for the reconstruction of energy and angles. Energy was finally calibrated to the peak position of Bhabha event for each fill. The reconstruction of $\theta$ and $\phi$ was done by taking into account the beam spot position defined for each run. Run-time corrections on the positions of tagged particles due to the incident angles of the beams were defined for each run or fill as well, and applied to reject the background from Bhabha events and off-momentum electrons. Invariant mass, which is reconstructed in small angle approximations from the energy measurements of the tagged particles, is shown in Fig. 7. It can be seen that the acceptable range of invariant masses that can be used for the total $\gamma \gamma$ cross-section measurements is expanded 


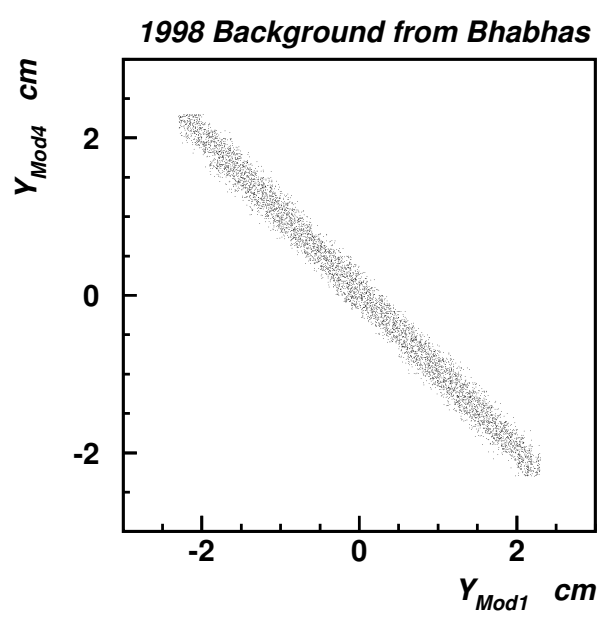

Figure 4: Y coordinates measured by the VSAT modules for both scattered leptons. Normal Bhabha events.

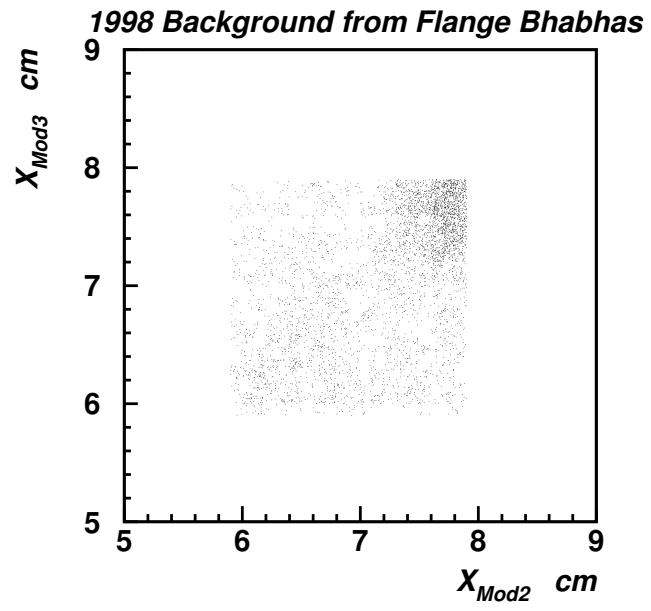

Figure 5: X coordinates measured by the VSAT modules for both scattered leptons. "Flange" Bhabha events. 


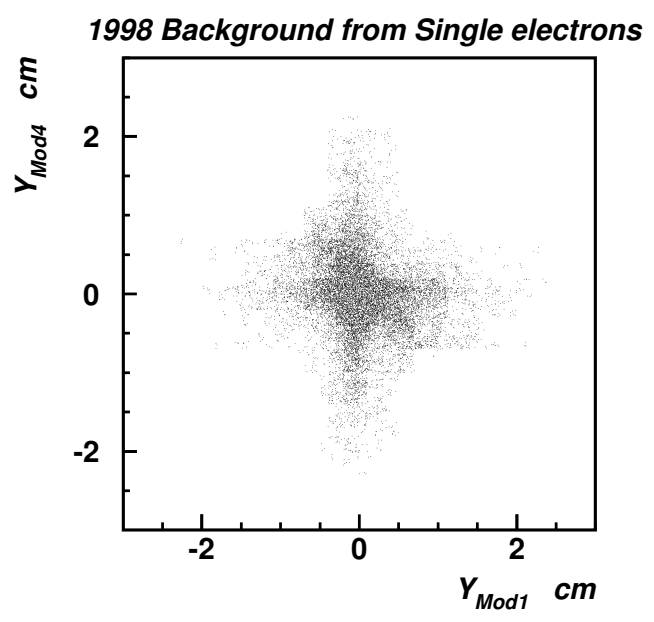

Figure 6: Y coordinates measured by the VSAT modules for both scattered leptons. False Bhabha events.

up to $\simeq 125 \mathrm{GeV}$. Momentum transfer squared $Q^{2}$ reconstructed from the energy and $\theta$ of tagged particles is shown in Fig. 8. It is clearly seen that all statistics are below $0.8 \mathrm{GeV}^{2} / c^{2}$, with a maximum at around $0.2 \mathrm{GeV}^{2} / c^{2}$. Several interesting features can be easily derived from both distributions. The QPM contribution is completely negligible. More importantly the main contribution to the cross-section comes from the RPC component, whereas at LEP1 it mainly came from the VDM while the RPC contributed $\simeq 22 \%$. Thus it becomes possible to study this relatively new process of hard scattering of the photon constituents more effectively. Theoretical predictions [10] suggest that the behaviour of the cross-section versus the difference of azimuthal angles of tagged particles could usefully be studied. The special acceptance of the VSAT, shown in Fig. 9, makes it possible to obtain the results in the restricted ranges of $\Delta \phi$ where an agreement between data and events simulated in the TWOGAM can be seen.

Taking into account the general agreement between the data and Monte Carlo it becomes possible to roughly estimate a total effective $\gamma \gamma$ cross-section using the improved photon flux calculations [11]. Results are shown in Table 1. with only statistical errors presented.

Table 1: Effective total $\gamma \gamma$ hadronic cross-section

\begin{tabular}{|c|c|c|}
\hline$\sqrt{s} G e V$ & No of events & Total $\sigma_{\text {Tot }}^{\gamma \gamma} n b$ \\
\hline 75 & 19 & $410 \pm 94$ \\
\hline 85 & 16 & $350 \pm 88$ \\
\hline 95 & 16 & $345 \pm 86$ \\
\hline
\end{tabular}




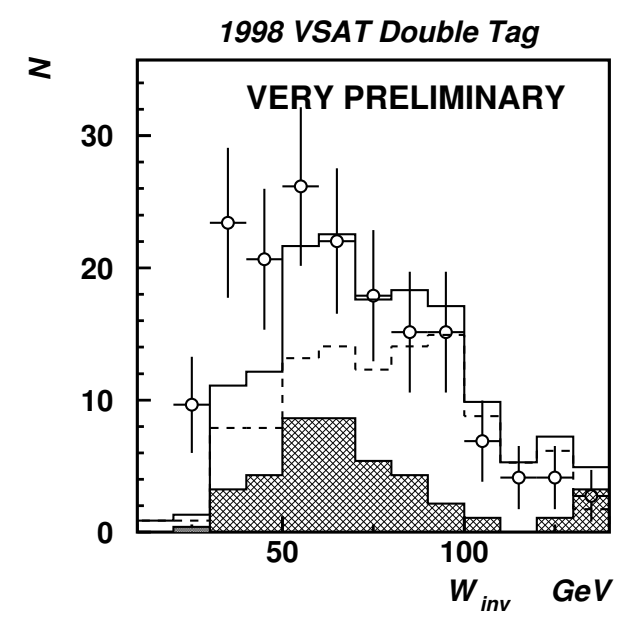

Figure 7: Invariant mass reconstructed. Points are the data, solid line is the full $\mathrm{VDM}+\mathrm{QPM}+\mathrm{RPC}$, dotted line is the RPC, hatched histogram is the VDM and filled histogram (only one bin is visible) is the QPM part.

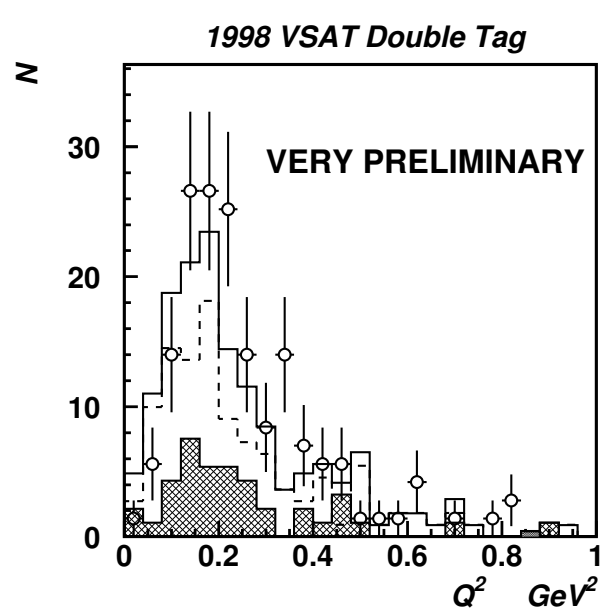

Figure 8: Momentum transfer squared. Points are the data, solid line is the full $\mathrm{VDM}+\mathrm{QPM}+\mathrm{RPC}$, dotted line is the RPC, hatched histogram is the VDM and filled histogram (only one bin is visible) is the QPM part. 


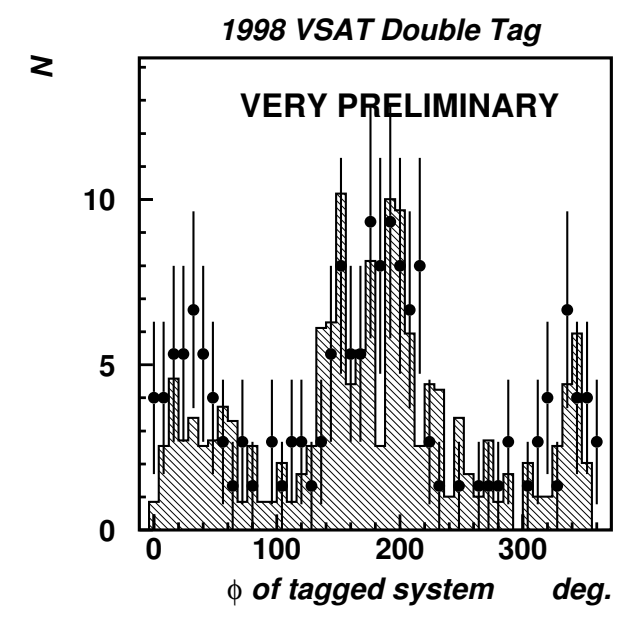

Figure 9: Azimuthal angle $\phi$ reconstructed for the tagged system. Points are the data, the histogram is Monte Carlo prediction.

\section{CONCLUSIONS}

Double tagged hadronic events produced in two-photon collisions at LEP2 at very low momentum transfer $Q^{2}$ have been studied for the first time. Reasonable agreement with Monte Carlo predictions was shown for such events after background rejection. The effective total $\gamma \gamma$ cross-section was estimated for $\gamma \gamma$ centre of mass energy up to 120 $\mathrm{GeV}$. At higher centre-of-mass energy $\sqrt{s} \simeq 189 \mathrm{GeV}$ the relative contribution from the QCD-RPC plays a dominant role $(\simeq 70 \%)$. Thus, new ways of making precise checks of this component of the model will open up when higher statistics became available from the last two years of LEP2 operation.

\section{References}

[1] P. Aurenche et al., Physics at LEP2, eds. G. Altarelli, T. Sjöstrand and F. Zwirner, CERN 96-01 (1996) 291.

[2] N. Zimin, Proc. Workshop on Photon Interactions and the Photon Structure, eds. G. Jarlskog and T. Sjöstrand, Lund (1998) 117.

[3] S. Nova, A. Olshevski and T. Todorov, DELPHI Note 90-35 (1990).

[4] DELPHI Coll., P. Abreu et al., Z. Phys. C 62, (1994) 357.

[5] DELPHI Coll., P. Abreu et al., Phys. Lett. B342 (1995) 402.

[6] N. Zimin, Proc. Photon '97, Egmond aan Zee, eds. A. Buijs and F. Erne., World Scientific, Singapore, (1997) 74.

[7] P. Abreu et al. (DELPHI Coll.), NIM A378 (1996) 57. 
[8] S. Almehed et, NIM, A305 (1991) 320.

[9] E. Bravin et al., Proc. DIPAC 97, Frascati, (1997) LNF-97-048-IR, eds. A. Ghigo, M. Giabbai and G. Possanza.

[10] V. M. Budnev et al., Phys. Rep. 15, (1975) 181 (see pages 226, 244-245).

[11] G. Schuler, CERN-TH/96-297 (1996) 